\title{
Effects of Dimethyl Sulfoxide on Neuronal Response Char- acteristics in Deep Layers of Rat Barrel Cortex
}

\author{
Narjes Soltani ${ }^{1}$, Elham Mohammadi ${ }^{1}$, Mohammad Allahtavakoli ${ }^{1}$, Ali Shamsizadeh ${ }^{{ }^{*}}$, Ali Roohbakhsh ${ }^{2}$, Abbas Haghparast \\ 1. Physiology-Pharmacology Research Center, Rafsanjan University of Medical Sciences, Rafsanjan, Iran. \\ 2. Pharmaceutical Research Center, School of Pharmacy, Mashhad University of Medical Sciences, Mashhad, Iran. \\ 3. Neuroscience Research Center, Shahid Beheshti University of Medical Sciences, Tehran, Iran.
}

cttation: Soltani, N., Mohammadi, E., Allahtavakoli, M., Shamsizadeh, A., Roohbakhsh, A., \& Haghparast, A. (2016). Effects of dimethyl sulfoxide on neuronal response characteristics in deep layers of rat barrel cortex. Basic and Clinical Neuroscience, 7(3), 213-220.

http://dx.doi.org/10.15412/J.BCN.03070306

: http://dx.doi.org/10.15412/J.BCN.03070306

Article info:

Received: 21 April 2015

First Revision: 13 June 2015

Accepted: 11 September 2015
Key Words:

DMSO,

Electrophysiology,

Somatosensory cortex, Rats

\begin{abstract}
A B S T R A C T
Introduction: Dimethyl sulfoxide (DMSO) is a chemical often used as a solvent for waterinsoluble drugs. In this study, we evaluated the effect of intracerebroventricular (ICV) administration of DMSO on neural response characteristics (in 1200-1500 $\mu \mathrm{m}$ depth) of the rat barrel cortex.
\end{abstract}

Methods: DMSO solution was prepared in $10 \% \mathrm{v} / \mathrm{v}$ concentration and injected into the lateral ventricle of rats. Neuronal spontaneous activity and neuronal responses to deflection of the principal whisker (PW) and adjacent whisker (AW) were recorded in barrel cortex. A condition test ratio (CTR) was used to measure inhibitory receptive fields in barrel cortex.

Results: The results showed that both PW and AW evoked ON and OFF responses, neuronal spontaneous activity and inhibitory receptive fields did not change following ICV administration of DMSO.

Conclusion: Results of this study suggest that acute ICV administration of 10\% DMSO did not modulate the electrophysiological characteristics of neurons in the 1 deep ayers of rat barrel cortex.

\section{Introduction}

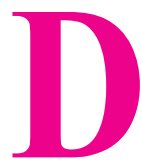

imethyl sulfoxide (DMSO) is a chemical with formula $\left(\mathrm{CH}_{3}\right)$ 2SO. It is widely used in neuroscience research as a solvent for various pharmacological agents in both in vitro and in vivo studies ( $\mathrm{Lu} \&$ Mattson, 2001). The most frequent concentration of DMSO which is generally used in neuroscience (in vivo) studies is $10 \% \mathrm{v} / \mathrm{v}$ (Amiresmaili, Shamsizadeh, Allahtavakoli,
Pourshanazari, \& Roohbakhsh, 2014; Roohbakhsh, Keshavarz, Hasanein, Rezvani, \& Moghaddam, 2009). There are many reports about the biological effects of DMSO, especially in neuroscience. For example, it was reported that DMSO blocks $\mathrm{Na}^{+}, \mathrm{K}^{+}$and $\mathrm{Ca}^{++}$currents (Morley \& Whitfield, 1993; Ogura, Shuba, \& McDonald, 1995; Rosenblum, Wei, \& Kontos, 2001). Also, DMSO shifts the sodium inactivation curve for neuroblastoma-glioma hybrid NG108-15 cells to more negative voltages (Jourdon, Berwald-Netter, \& Dubois, 1986). In aplysia gan-

\footnotetext{
* Corresponding Author:

Ali Shamsizadeh, $P h D$

Address: Physiology-Pharmacology Research Center, Rafsanjan University of Medical Sciences, Rafsanjan, Iran.

Tel: +98 (34) 34339660

E-mail:alishamsy@gmail.com
} 
glion cells, DMSO depresses the increase in membrane permeability induced by acetylcholine, glutamate, and GABA (Sawada \& Sato, 1975). DMSO also suppresses NMDA and AMPA currents in hippocampal neurons and decreases NMDA receptors activation in these cells (Lu \& Mattson, 2001). Most of the previous studies about the biological effects of DMSO were carried out using in vitro studies and less is known about in vivo effects of DMSO, especially in the cortex.

Barrel cortex is a discrete cortical representation of whiskers of the rodent primary somatosensory cortex. Many studies have shown that there is a one to one correspondence between whiskers and cortical barrels. Neurons in each barrel give the strongest response to one single whisker (i.e. their principal) and weaker response to several adjacent whiskers (AWs) (Diamond, Armstrong-James, \& Ebner, 1993; Rema, Armstrong-James, \& Ebner, 2003). In addition, cortical neuronal receptive fields have both excitatory and inhibitory components (Shimegi, Ichikawa, Akasaki, \& Sato, 1999). These features make the whiskerbarrel system a good model for studying the neuronal electrophysiological characteristics in the cortex.

In this study, we evaluated the effect of intracerebroventricular (ICV) administration of 10\% DMSO on neural response characteristics to natural stimuli by using vibrissae pathway.

\section{Methods}

\subsection{Animals}

This study was carried out on 10 male Wistar rats, weighing $230-260 \mathrm{~g}$. The rats were housed in standard cages with a 12:12 hour light/dark cycle. The laboratory temperature was set at $23 \pm 2.0^{\circ} \mathrm{C}$. Food and water was available ad libitum. All experimental procedures were carried out in accordance with the guidelines for the care and use of laboratory animals observed at Rafsanjan University of Medical Sciences and the European Communities Council Directive of 24 November 1986 (86/609/EEC).

\subsection{Extracellular single unit recording}

As reported previously (Shamsizadeh, Sheibani, Arabzadeh, Afarinesh, Farazifard, Noorbakhsh, et al. 2007), extracellular single unit recording were carried out in right barrel cortex. For this purpose, animals were firstly anesthetized by administration of $1.5 \mathrm{~g} / \mathrm{kg} / \mathrm{i} . \mathrm{p}$ urethane. Then, the right somatosensory cortex was exposed by a 4-mm craniotomy. Tungsten microelectrodes (FHC, Bowdoinham, ME) were inserted (by a micro- manipulator) through the barrel cortex at the depth of $1000-1500 \mu \mathrm{m}$ from the skull surface. The electrodes were oriented perpendicular to the cortical surface. The data collected from tungsten microelectrodes were amplified and digitized by homemade electromodule (Electrophysiology recording system; ScienceBeam Co., Tehran, Iran).

Single neuron action potentials were isolated online using an amplitude window discriminator provided by eProbe software (Recording and analyzing software solution; ScienceBeam Co., Tehran, Iran) and the accuracy of isolation was judged by spike waveform and amplitude criteria. Interspike intervals were calculated in the first 50 $\mathrm{ms}$ after deflection of the spared principal whisker to verify the precision of single unit isolation. The average interspike intervals were significantly above $1 \mathrm{~ms}$ (1-tailed t-test, all $\mathrm{P}<0.05$ ) for all recorded units. Similar to the previous studies (Armstrong-James \& Fox, 1987; Erchova \& Diamond, 2004; Wallace, Glazewski, Liming, \& Fox, 2001), neurons collected from depths of 1000-1500 $\mu \mathrm{m}$ were located within layers $\mathrm{V}$ and VI of barrel cortex.

To ensure that recording was made in barrel cortex, the recording electrode was first inserted at a depth of 500-800 $\mu \mathrm{m}$ from the skull then the PW was stimulated and the latency of neuronal response to PW deflection was measured. In all experiments the response latency was below $10 \mathrm{~ms}$.

During the recording time, the cortical surface was covered by $3 \%$ agar solution. Also depth of anesthesia was held at the level which characterized by the absence of spontaneous movement and overt whisking or whisker tremor. The respiration was regular (80-100 breaths/ $\min$ ) throughout the anesthesia period. Sluggish hind limb withdrawal reflex and also corneal and eyelid reflex were present during anesthesia. Whenever necessary, additional doses of urethane ( $10 \%$ induction dose) were used to maintain the level of anesthesia. Rectal temperature was maintained at $37^{\circ} \mathrm{C}$ by a servo-controlled heating blanket (Harvard Apparatus, Eden Bridge, UK).

\subsection{Whisker stimulation}

Fine mechanical displacement was produced by two tiny speakers triggered by trapezoid shape wave form by electromodule (Electrophysiology recording system, ScienceBeam Co., Tehran, Iran). Speakers were connected to a thin glass tube (an internal diameter of 0.69 $\mathrm{mm})$. By delivering computer-gated currents, speakers produced ramp and hold movements with an amplitude of $500 \mu \mathrm{m}$, onset and offset rise times of $5 \mathrm{~ms}$, and a 
duration of $200 \mathrm{~ms}$. The PW and AWs were cut $10 \mathrm{~mm}$ from the base and inserted inside the tubes.

\subsection{Recording procedures}

$\mathrm{ON}$ and OFF responses were elicited following whisker deflection. The ON response was elicited when the stimulator began to move and the OFF response was elicited when the stimulator returned to its resting position (Shamsizadeh et al., 2007). A conditioning test paradigm were used to study the neuronal responses to combined stimulation of 2 vibrissae (Simons, 1985). During the conditioning paradigm adjacent and principal whiskers were deflected in succession at $30 \mathrm{~ms}$ ISIs (interstimulus interval). Each of these 3 stimulation patterns ( 2 single PW and AW deflection and 1 combined AW-PW deflections) was repeated 50 times in a pseudorandom order. Each stimulation pattern lasted $1 \mathrm{~s}$. Electromodule software was used both for the stimulus delivery and the storage of spike times. Peristimulus time histograms were produced in response to each of the 3 deflection patterns. Magnitudes of the $\mathrm{ON}$ and OFF responses were calculated by counting the number of spikes from 5 to $35 \mathrm{~ms}$ after the onset of each movement. Whiskers interaction were quantified with a condition-test ratio (CTR), which has been previously used in the study of sensory integration (Ego-Stengel, Mello e Souza, Jacob, \& Shulz, 2005; Simons \& Carvell, 1989).

This ratio measures the effect of the conditioning stimulus (the AW deflection) on the response of the unit to the test stimulus (the PW deflection). The observed response was considered as the magnitude of response to PW stimulation in combined in combine deflection pattern. The expected response was estimated by summing the response to deflection of the PW alone with the appropriate late part of the response to the AW deflection alone. The CTR was defined as the ratio of the observed to the expected responses (Simons \& Carvell, 1989). A ratio of less than 1 indicates response suppression whereas a ratio of more than 1 indicates response facilitation.

\subsection{Drugs}

DMSO was purchased from Merck Company. One milliliter DMSO was diluted with $9 \mathrm{~mL}$ distilled water to reach the concentration of $10 \% \mathrm{v} / \mathrm{v}$. Then, $5 \mu \mathrm{L}$ of DMSO $10 \%$ was injected slowly (within $3 \mathrm{~min}$ ) to the left lateral ventricle by Hamilton syringe. The stereotaxic coordinates for the left to right ventricle were $1 \mathrm{~mm}$ posterior to bregma, $1.5 \mathrm{~mm}$ lateral to the midline, terminating $3.5 \mathrm{~mm}$ ventral of the dorsal surface of the skull according to Paxinos and Watson's rat brain atlas (Paxinos \& Watson, 2006). DMSO injection was done twice.
In the first time, we measured spontaneous activity for 30 minutes after drug injection. Second drug injection was done $1 \mathrm{~h}$ later and we measured neuronal responses to whiskers deflections $15 \mathrm{~min}$ after second injection.

\subsection{Statistical analysis}

Recording was done before and 15 minutes after ICV administration of DMSO. The recorded signals were imported to eSorter (Spike Sorting Software toolbox, ScienceBeam Co., Tehran, Iran) and spike potentials peak and valley amplitude features were extracted and classified by k-means clustering offline. The classified spikes were further analyzed for $\mathrm{ON}$ and $\mathrm{OFF}$ responses by eSpike. A $\mathrm{P}$-value less than 0.05 was considered significant. Paired ttest was used to compare ON and OFF responses and also CTR ratio before and after DMSO administration.

\section{Results}

\subsection{Effect of DMSO on neuronal spontaneous ac- tivity}

For studying the effect of DMSO on neuronal spontaneous activity, we recorded spontaneous activity for $5 \mathrm{~min}$ utes. Then, DMSO was administered through ICV and the spontaneous activity was recorded for 30 minutes. Figure 1 shows population time histogram of neuronal spontaneous responses before and after DMSO administration. Frequencies of spikes were $47.9 \pm 8.8$ and $59 \pm 8.6$ $\mathrm{Hz}$ before and after DMSO administration respectively which were not different significantly $(\mathrm{P}>0.05)$.

\subsection{Effect of DMSO on neuronal excitatory recep- tive fields}

For studying excitatory receptive fields, we measured neuronal $\mathrm{ON}$ and OFF responses to deflection of $\mathrm{PW}$ and AW before and 15 minutes after DMSO administration (see methods). Figure 2 shows samples of neuronal response to deflection of PW (A \& B) and AW (C \& D) before $(\mathrm{A} \& \mathrm{C})$ and after $(\mathrm{B} \& \mathrm{D})$ administration of DMSO.

Neuronal ON and OFF responses magnitude to PW deflection were not affected following ICV administration of DMSO (all $\mathrm{P}>0.05$ ) (Table 1). Similarly, ON and OFF response magnitudes to deflection of AW were not affected following ICV administration of DMSO (all $\mathrm{P}>0.05$ ) (Table 2).

3.3. Effect of DMSO on temporal profile of response integration

To investigate vibrissae interactions, a conditioningtesting paradigm was used in which AW deflection 
A)

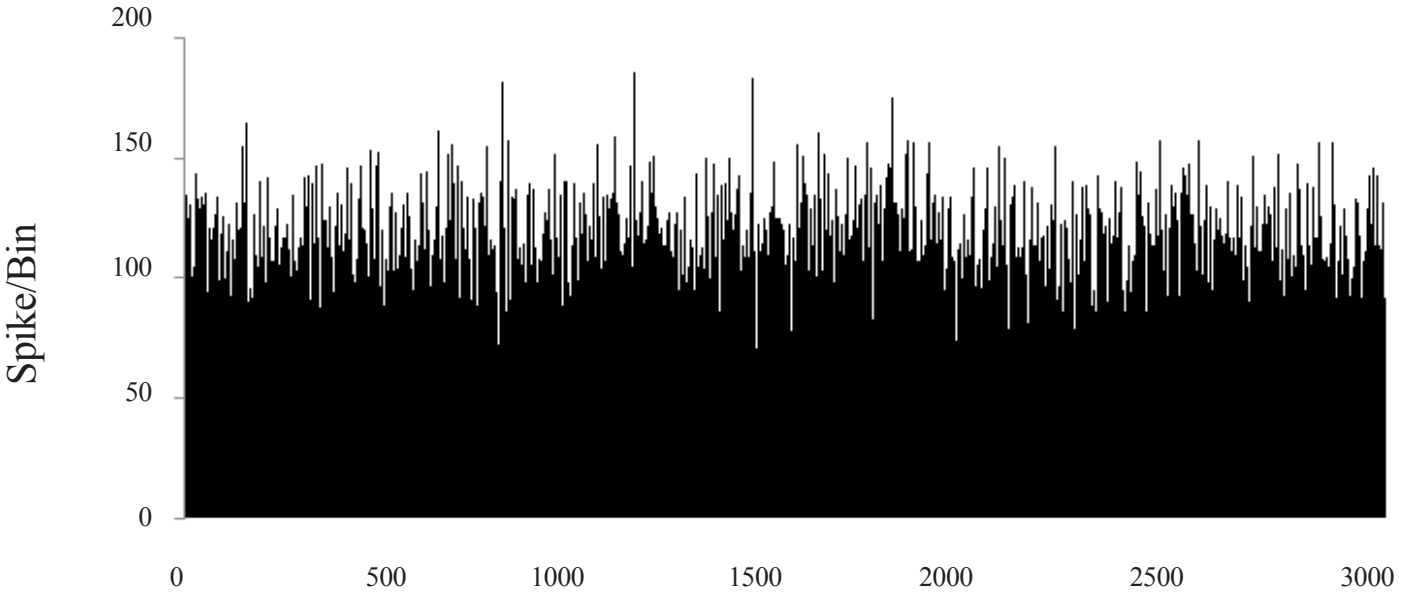

Time $(\mathrm{ms})$

B)

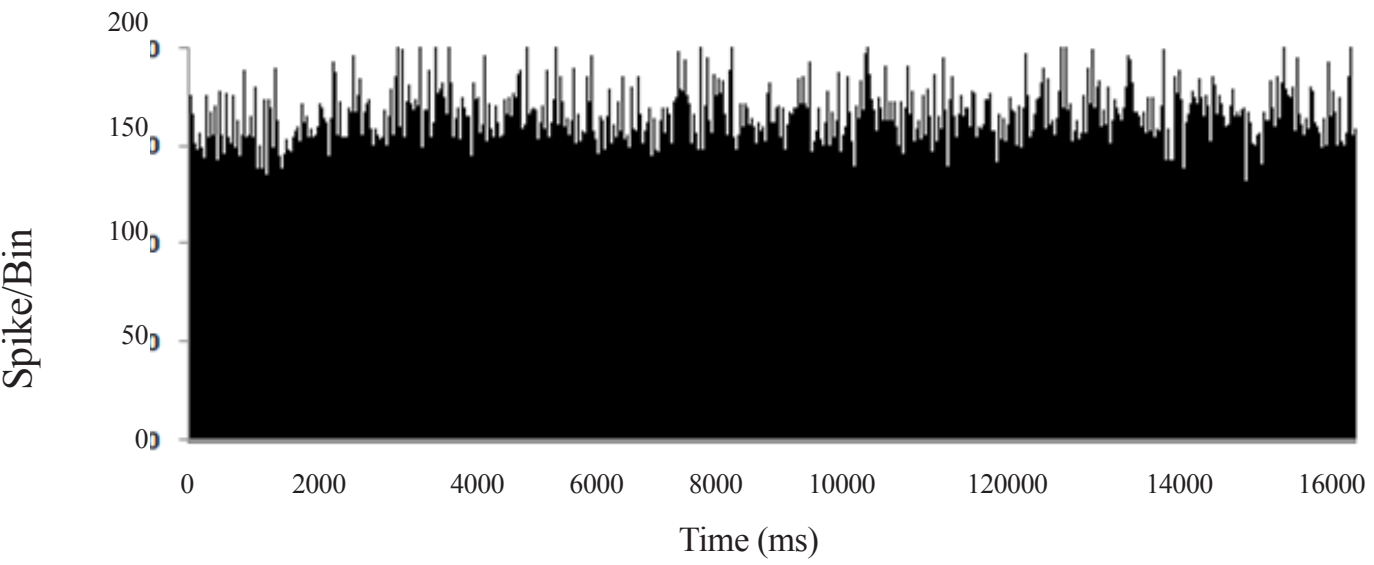

NEUR:SCIENCE

Figure 1. Population time histogram of spontaneous responses before and after DMSO administration. In panel A, recording was done for 5 minutes before drug administration. In panel B, recording was done for 30 minutes after DMSO administration.

was preceded PW deflection at the interstimulus interval (ISI) of $30 \mathrm{~ms}$. The strengths of these interactions were quantified applying a condition-test ratio (CTR) measurement (see methods). CTR ratio for both $\mathrm{ON}$ and OFF responses were not affected following DMSO administration (all $\mathrm{P}>0.05$ ) (Figure 3 ).

\section{Discussion}

In this study, we investigated the effect of ICV administration of DMSO on neuronal spontaneous activity and neuronal response properties to natural stimuli by employing vibrissae pathway model in rats. Our results revealed that neuronal spontaneous activity and neuronal response properties to deflection principal and adjacent whiskers and also temporal aspects of response integration did not change following 10\% DMSO injection.

DMSO is an organic solvent that is used as a vehicle owning to its miscibility with water and ability to increase membrane permeability, thereby facilitating the absorption of compounds that are otherwise insoluble in water. However, some studies suggest that DMSO may be toxic or cause nonspecific effects, casting doubt on whether DMSO is indeed a suitable vehicle for the administration of these substances in vivo.

The effects of DMSO is different and depend on some factors such as concentration and type of experiment and cells. In cell culture, DMSO has been found to directly influence cell excitability by altering $\mathrm{Ca}^{+2}, \mathrm{~K}^{+}, \mathrm{Na}^{+}$, or 
A

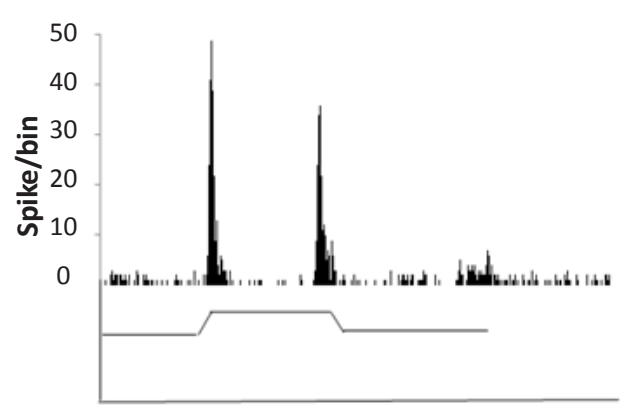

C

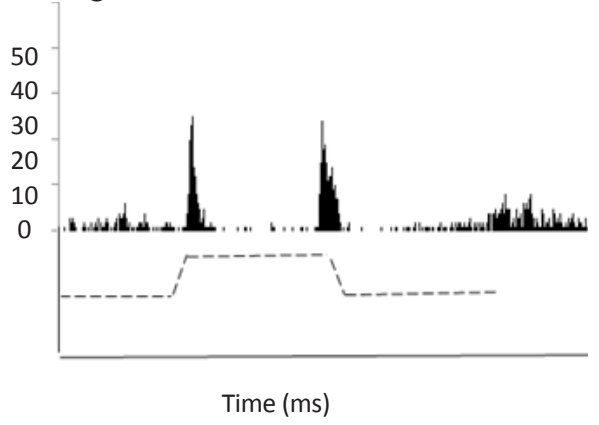

B

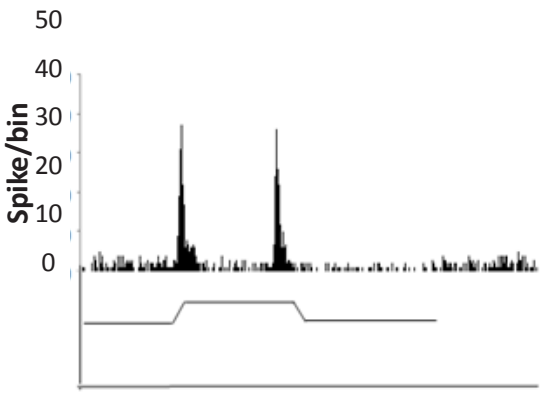

D

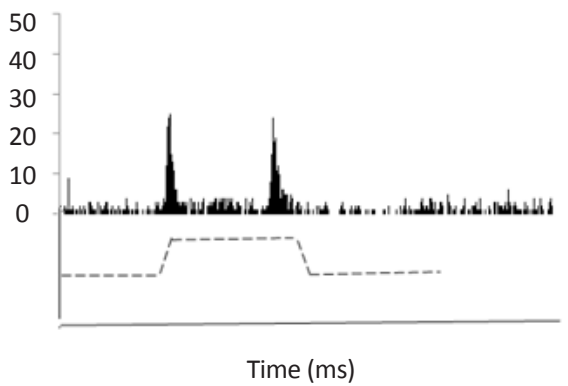

NEUR:SCIENCE

Figure 2. A typical example of peristimulus time histograms (bin=1 ms) showing ON and OFF neuronal responses to PW and AW movement. A \& B are responses to deflection of PW before and after DMSO administration, respectively. C \& D are responses to deflection of AW before and after DMSO administration, respectively.

Table 1. Neuronal ON and OFF response magnitude to deflection of "principle whisker" before and 15 minutes after ICV administration of DMSO.

\begin{tabular}{|c|c|c|}
\hline Time of recording & ON response & Off response \\
\hline Before & $14.56 \pm 3.1$ & $10.84 \pm 2.7$ \\
\hline After & $13.95 \pm 1.9$ & $12.53 \pm 1.8$ \\
\hline
\end{tabular}

Data are shown as the mean of neural spikes/bin/50 stimulation. Bin size is $1 \mathrm{~ms}$.

NEUR:SCIENCE

Table 2. Neuronal ON and OFF response magnitude to deflection of "adjacent whisker" before and 15 minutes after ICV administration of DMSO.

\begin{tabular}{|c|c|c|}
\hline Time of recording & ON response & Off response \\
\hline Before & $10.35 \pm 2.5$ & $9.96 \pm 2.7$ \\
\hline After & $9.72 \pm 9.7$ & $9.62 \pm 1.5$ \\
\hline
\end{tabular}

Data are shown as the mean of neural spikes/bin/50 stimulation. Bin size is $1 \mathrm{~ms}$.

NEUR:SCIENCE

$\mathrm{Cl}^{-}$currents (Connors \& Gutnick, 1990; Morley \& Whitfield, 1993; Nakahiro, Arakawa, Narahashi, Ukai, Kato, Nishinuma, \& Nishimura, 1992; OguraOgura, Shuba, \& McDonald, (1995). Lu and Mattson (2001) reported that a DMSO concentration of $10 \%$ decreased the number of viable neurons in primary hippocampal cell cultures (Lu \&
Mattson, 2001). In addition, they reported that DMSO suppressed NMDA and AMPA currents and decreased NMDA receptors activation in these cultured neurons ( $\mathrm{Lu} \&$ Mattson, 2001). On the other hand, DMSO blocks GABA-induced current in rat dorsal ganglion neurons (Nakahiro et al., 1992). 


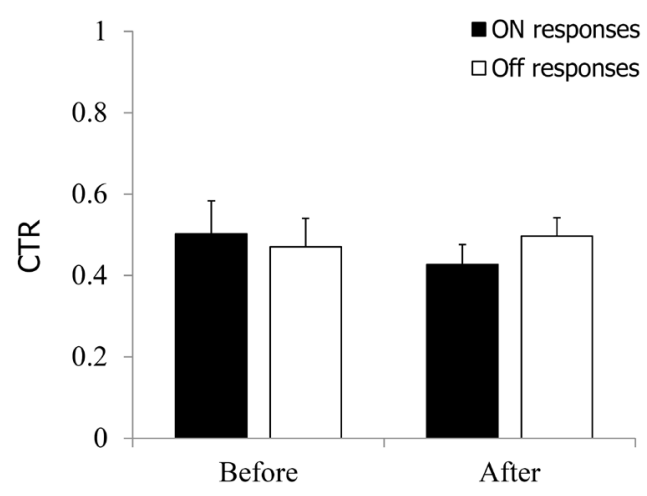

NEUR SCIENCE

Figure 3. CTR for ON and OFF responses before and after DMSO injection. Graphs plot the proportional change in CTR as a function of interdeflection time interval. The value of CTR $>1$ indicates facilitation, while $<1$ means inhibitory effect of the first whisker deflection on response to second whisker deflection. Error bars represent S.E.M.

Some studies reported that there are differences between short-term and long-term effects of DMSO. Fossum Lisowski, Macey, Ingram and Morgan (2008) reported that acute administration of DMSO did not cause antinociception when administrated into the periaqueductal gray alone, whereas repeated administration of DMSO reduced the potency of subsequent morphine administration (Fossum, Lisowski, Macey, Ingram, \& Morgan, 2008).

Many studies imply that the electrophysiological characteristics of neurons in cortical circuits are regulated by neuromodulatory systems (Allene \& Cossart, 2010; Khazipov \& Luhmann, 2006) such as glutamatergic and GABAergic neurons (Lopez-Bendito \& Molnar, 2003). With regard to our finding that DMSO has no effect on electrophysiological properties of neurons in the barrel cortex, we speculate that this condition may be due to dual effects of DMSO on suppressing both excitatory and inhibitory networks in the barrel cortex. In line with these findings, Cavas and associates (2005) reported that DMSO in $5 \%$ or $10 \%$ concentrations has no effect on EEG in rats but DMSO in $15 \%$ or $20 \%$ concentrations, increases light slow wave sleep and decreases deep slow wave sleeps (Cavas, Beltran, \& Navarro, 2005).

In this study, we used only $10 \%$ DMSO, which is widely used as solvent for ICV administration of water insoluble drugs. However, we suggest that the effect of other concentrations of DMSO on neural response characteristics be evaluated too. Also, in this study, neurons were collected from depths of 1000-1500 $\mu \mathrm{m}$, located within layers V and VI of barrel cortex. As neurons in differ- ent layers of cortex have different electrophysiological properties (Connors \& Gutnick, 1990), we need to record from other layers of cortex to have a better interpretation for the effects of DMSO on electrophysiological properties of neurons in the cortex.

In conclusion, based on the results of this study that ICV administration of $10 \%$ DMSO not changing neuronal response properties in layers V and VI in the barrel cortex, $10 \%$ DMSO is potentially an ineffective solvent for ICV administrations of drugs.

\section{Acknowledgements}

The authors acknowledge Physiology-Pharmacology Research Center of Rafsanjan University of Medical Sciences for the financial support of this study.

\section{References}

Allene, C., \& Cossart, R. (2010). Early NMDA receptor-driven waves of activity in the developing neocortex: physiological or pathological network oscillations? Journal of Physiology, 588(1), 83-91. doi: 10.1113/jphysiol.2009.178798

Amiresmaili, S., Shamsizadeh, A., Allahtavakoli, M., Pourshanazari, A. A., \& Roohbakhsh, A. (2014). The effect of intra-ventral hippocampus administration of TRPV1 agonist and antagonist on spatial learning and memory in male rats. Pharmacological Reports, 66(1), 10-14. doi: 10.1016/j. pharep.2013.06.002 
Armstrong-James, M., \& Fox, K. (1987). Spatiotemporal convergence and divergence in the rat $\mathrm{S} 1$ "barrel" cortex. Journal of Comparative Neurology, 263(2), 265-281. doi: 10.1002/ cne. 902630209

Cavas, M., Beltran, D., \& Navarro, J. F. (2005). Behavioural effects of dimethyl sulfoxide (DMSO): Changes in sleep architecture in rats. Toxicology Letters, 157(3), 221-232. doi: 10.1016/j.toxlet.2005.02.003

Connors, B. W., \& Gutnick, M. J. (1990). Intrinsic firing patterns of diverse neocortical neurons. Trends in Neurosciences, 13(3), 99-104.

Diamond, M. E., Armstrong-James, M., \& Ebner, F. F. (1993). Experience-dependent plasticity in adult rat barrel cortex. Proceedings of National Academy of Sciences of United States, 90(5), 2082-2086.

Ego-Stengel, V., Mello Souza, T., Jacob, V., \& Shulz, D. E. (2005). Spatiotemporal characteristics of neuronal sensory integration in the barrel cortex of the rat. Journal of Neurophysiology, 93(3), 1450-1467. doi: 10.1152/jn.00912.2004.

Erchova, I. A., \& Diamond, M. E. (2004). Rapid fluctuations in rat barrel cortex plasticity. Journal of Neuroscience, 24(26), 5931-5941. doi: 10.1523/jneurosci.1202-04.2004

Fossum, E. N., Lisowski, M. J., Macey, T. A., Ingram, S. L., \& Morgan, M. M. (2008). Microinjection of the vehicle dimethyl sulfoxide (DMSO) into the periaqueductal gray modulates morphine antinociception. Brain Research, 1204, 53-58. doi: 10.1016/j.brainres.2008.02.022

Jourdon, P., Berwald-Netter, Y., \& Dubois, J. M. (1986). Effects of dimethylsulfoxide on membrane currents of neuroblastoma x glioma hybrid cell. Biochimica et Biophysica Acta, 856(2), $399-402$

Khazipov, R., \& Luhmann, H. J. (2006). Early patterns of electrical activity in the developing cerebral cortex of humans and rodents. Trends in Neurosciences, 29(7), 414-418. doi: 10.1016/j. tins.2006.05.007

Lopez-Bendito, G., \& Molnar, Z. (2003). Thalamocortical development: how are we going to get there? Nature Reviews Neuroscience, 4(4), 276-289. doi: 10.1038/nrn1075

Lu, C., \& Mattson, M. P. (2001). Dimethyl sulfoxide suppresses NMDA-and AMPA-induced ion currents and calcium influx and protects against excitotoxic death in hippocampal neurons. Experimental Neurology, 170(1), 180-185. doi: 10.1006/ exnr.2001.7686

Morley, P., \& Whitfield, J. F. (1993). The differentiation inducer, dimethyl sulfoxide, transiently increases the intracellular calcium ion concentration in various cell types. Journal of Cellular Physiology, 156(2), 219-225. doi: 10.1002/jcp.1041560202

Nakahiro, M., Arakawa, O., Narahashi, T., Ukai, S., Kato, Y., Nishinuma, K., et al. (1992). Dimethyl sulfoxide (DMSO) blocks GABA-induced current in rat dorsal root ganglion neurons. Neuroscience Letters, 138(1), 5-8.

Ogura, T., Shuba, L. M., \& McDonald, T. F. (1995). Action potentials, ionic currents and cell water in guinea pig ventricular preparations exposed to dimethyl sulfoxide. Journal of Pharmacology and Experimental Therapeutics, 273(3), 1273-1286.
Paxinos, G., \& Watson, C. (2006). The rat brain in stereotaxic coordinates: Hard cover edition (6th ed.). San Diego, Elsevier: Academic Press.

Rema, V., Armstrong-James, M., \& Ebner, F. F. (2003). Experience-dependent plasticity is impaired in adult rat barrel cortex after whiskers are unused in early postnatal life. Journal of Neuroscience, 23(1), 358-366.

Roohbakhsh, A., Keshavarz, S., Hasanein, P., Rezvani, M. E., \& Moghaddam, A. H. (2009). Role of endocannabinoid system in the ventral hippocampus of rats in the modulation of anxietylike behaviours. Basic \& Clinical Pharmacology \& Toxicology, 105(5), 333-338. doi: 10.1111/j.1742-7843.2009.00449.x

Rosenblum, W. I., Wei, E. P., \& Kontos, H. A. (2001). Dimethylsulfoxide and ethanol, commonly used diluents, prevent dilation of pial arterioles by openers of K(ATP) ion channels. European Journal of Pharmacology, 430(1), 101-106.

Sawada, M., \& Sato, M. (1975). The effect of dimethyl sulfoxide on the neuronal excitability and cholinergic transmission in Aplysia ganglion cells. Annals of the New York Academy of Sciences, 243(1), 337-357.

Shamsizadeh, A., Sheibani, V., Arabzadeh, S., Afarinesh, M. R., Farazifard, R., Noorbakhsh, S. M., et al. (2007). Single whisker experience started on postnatal days 0,5 or 8 changes temporal characteristics of response integration in layers IV and V of rat barrel cortex neurons. Brain Research Bulletin, 74(1-3), 29-36. doi: 10.1016/j.brainresbull.2007.04.010

Shimegi, S., Ichikawa, T., Akasaki, T., \& Sato, H. (1999). Temporal characteristics of response integration evoked by multiple whisker stimulations in the barrel cortex of rats. Journal of Neuroscience, 19(22), 10164-10175.

Simons, D. J. (1985). Temporal and spatial integration in the rat SI vibrissa cortex. Journal of Neurophysiology, 54(3), 615-635.

Simons, D. J., \& Carvell, G. E. (1989). Thalamocortical response transformation in the rat vibrissa/barrel system. Journal of Neurophysiology, 61(2), 311-330.

Wallace, H., Glazewski, S., Liming, K., \& Fox, K. (2001). The role of cortical activity in experience-dependent potentiation and depression of sensory responses in rat barrel cortex. Journal of Neuroscience, 21(11), 3881-3894. 
